\title{
Yash Scoring System in the Diagnosis of Acute Appendicitis
}

\author{
Yeshwant Lamture' ${ }^{1}$, Rahul Y. Netragaonkar², Varsha Gajbhiye ${ }^{3}$, Ranjeet Ambad ${ }^{4}$ \\ ${ }^{1}$ Professor, Dept. of General Surgery, J.N.M.C. Sawangi (Meghe) Wardha, ${ }^{2}$ Associate Professor, Dept. of \\ Community Medicine, DVVPF'S Medical College, Ahmednagar, ${ }^{3}$ Asso. Professor in Pharmacology, Datta Meghe \\ Medical College. Nagpur, ${ }^{4}$ Associate Professor in Biochemistry, Datta Meghe Medical College. Nagpur
}

\begin{abstract}
Background: Acute appendicitis is amongst the most encountered emergencies seen in casualties requiring intervention. The incidence of negative laparotomy is $15 \%$ to $25 \%$ and is associated with notable morbidity. Scoring systems are useful and logical for distinguishing acute appendicitis from non-specific abdominal pain. Presently many scoring systems exist that aids in the diagnosis of acute appendicitis but still fail to decrease the rates of wrong diagnosis and the negative appendicectomy rate. This study has been undertaken to evaluate the efficacy of the newer Yash scoring system which has been found more effective for the Indian population in diagnosing acute appendicitis.
\end{abstract}

Method and Material: A prospective study of the Yash scoring system was done on 50 patients. The decision of an appendicectomy was taken by the consultant surgeon. The outcomes of the Yash scoring system in terms of sensitivity, specificity, positive predictive value, negative predictive value, and diagnostic accuracy were calculated.

Results: The Yash scoring system had sensitivity, specificity, PPV, NPV, and DA of 94.28\%, 93.33\%, $97.05 \%, 87.50 \%$, and $94 \%$ respectively.

Conclusion: The Yash scoring system was a good tool to diagnose acute appendicitis.

Keywords: Negative appendicectomy, C-reative protein, Hyperaesthesia in sherrens triangle, Blumberg sign.

\section{Introduction}

Acute appendicitis is amongst the many encountered emergencies seen in casualties requiring intervention. There is a $6 \%$ chance of appendicitis in the general population ${ }^{[1]}$. Though the mortality has declined from $26 \%$ to $1 \%$ with the dawn of broad-spectrum antibiotics and timely surgery, it remains 5 to $15 \%$ in the elderly ${ }^{[2]}$. The incidence of negative laparotomy is $15 \%$ to $25 \%$ and is associated with notable morbidity, the frequency being more in women of reproductive age group

\section{Corresponding Author:}

\section{Dr. Rahul Y. Netragaonkar}

Associate Professor, Dept. of Community Medicine, DVVPF`S Medical College, Ahmednagar e-mail: dryrlamture@yahoo.co.in (till 45\%) due to the commonness of inflammatory tubo-ovarian diseases, ectopic pregnancy, and other gynecological pathology ${ }^{[3]}$. The delay in diagnosis may lead to rupture of the appendix in $17 \%$ to $40 \%$ of cases mostly in extremes of age. In the adolescents and elderly, it is linked with dramatic complications like an intraabdominal abscess, wound infection, and ultimately death ${ }^{[4]}$. Thus, confirmation of acute appendicitis is very crucial to achieve lower morbidity and mortality rates.

The regular laboratory investigations of blood and urine are necessary. Leukocytosis is a convenient finding but is non-specific and may be missing in the elderly ${ }^{[5] .} \mathrm{C}$ - Reactive protein is elevated in almost all acute inflammatory conditions, hence is considered as a non-specific marker but its estimation guides a surgeon in reducing negative appendicectomies ${ }^{[6,7]}$. 
Magnetic resonance imaging (MRI) as an investigation is only taken typically in the pregnant population where radiation exposure is not warranted. It provides outstanding resolution and is highly efficient in diagnosing acute appendicitis. MRI has high sensitivity and specificity of $100 \%$ and $98 \%$ respectively. The PPV and NPV of MRI are $98 \%$ and $100 \%$ respectively. It is also operator-independent. The only issue is its higher cost, motion artifact, and complexity in reading MRI by non-radiologists with limited experience ${ }^{[6]}$.

Despite the use of all these imaging techniques, the incidence of negative appendicectomy is not decreasing. These unmerited operations have a complication rate of approximately $13 \%$, which is nearby to that of an inflamed appendix. Removal of a healthy appendix has a mortality of $0.65 \%$. Protracted clinical observations aiming to reduce undesired operations may mean a delay in operations in $28 \%$ of cases and considerable danger of perforation $^{[7,8,9] \text {. }}$

Scoring systems are useful and logical for distinguishing pain of acutely inflamed appendix from vague abdominal pain. Presently numerous scoring systems exist that aid in diagnosing acute appendicitis. However, these systems do not replace clinical acumen thereby just helping in the determination of acute appendicitis and aids in approaching a conclusion, whether a particular case should be operated or not, thus lowering the negative appendicectomy rate (NAR) ${ }^{[10,11]}$.

The study done by Lamture YR et al ${ }^{[12]}$ in India on the Yash scoring system reveals a sensitivity of $99.48 \%$, a specificity of $92.86 \%$, PPV of $99.48 \%$, and NPV of $92.86 \%$. Though the validity of the Yash scoring system is promising for the Indian population, its only disadvantage was in the mode of a single study.

Despite various scoring systems and developments in the diagnostic and imaging modalities, the diagnosis of appendicitis is in dilemma which fails to decrease the rates of wrong diagnosis and the negative appendicectomy rate. This sequentially increases the cost for diagnosis by the use of expensive radiological modalities like Computed Tomography and MRI thus causing a delay in the treatment leading to an increase in morbidity and mortality of the patients ${ }^{[13]}$

This non-randomized prospective study has been undertaken to evaluate the effectiveness of the newer Yash score which has been found more effective for the Indian population in diagnosing acute appendicitis.

\section{Method}

The present study was undertaken in the department of surgery, Jawaharlal Nehru Medical College, Wardha in collaboration with Datta Meghe Medical College Hingana, Nagpur, Datta Meghe Institute of medical science (DMIMS), Sawangi, Meghe, Wardha, Maharashtra India.

Study Design: Prospective non-randomized study.

Study Population: All patients of acute appendicitis with age $>15$ years and $<60$ years.

Study Duration: July 2018-July 2020.

Sample Size: 50 Patients

Inclusion Criteria: All patients with right lower quadrant pain and clinically diagnosed as acute appendicitis of age $>15$ years and $<60$ years.

\section{Exclusion Criteria:}

1. Patients with appendicular mass.

2. Patients of appendicitis with a known case of connective tissue disorder.

3. Patients with a past history of renal or ureteric stones and pelvic inflammatory disease.

4. Pregnant women.

Ethical clearance was obtained from the Ethics committee of Datta Meghe Institute of Medical Sciences (Deemed to be University) [Ref. No. DMIMS(DU)/ IEC/2018-19/7426].This prospective non-randomized study was conducted in Acharya Vinoba Bhave Rural Hospital, Sawangi. All the patients who fulfilled the eligibility criteria were subjected to routine hematological investigations, C-reactive protein, USG, and were scored based on the Yash scoring system.

\section{The components of the Yash scoring system are as follows:}

- Migration of pain to the right iliac fossa $=1$ point

- Nausea and vomiting $=1$ point

- $\quad$ Anorexia $=1$ point

- $\quad$ Right iliac fossa tenderness $=2$ points

- $\quad$ Rebound tenderness $=1$ point

- Hyperesthesia in Sherren's triangle $=1$ point

- $\quad$ Fever $=1$ point 
- White blood cell count $>10,000 \mathrm{mg} / \mathrm{dl}=2$ points

- C-reactive protein $(>15 \mathrm{mg} / \mathrm{dl})=3$ points

- Ultrasonography $=4$ points

A score of 7 or more is suggestive of acute appendicitis requiring surgery ${ }^{[12]}$.

The decision to operate was taken by a consultant surgeon by clinical diagnosis with help of other appropriate investigations in special circumstances. All patients underwent appendicectomy with prior consent and the specimen was subjected to histopathological examination for confirmation of diagnosis. The minimum criteria for acute appendicitis were the appearance of neutrophils in mucosa, submucosa, and lamina propria ${ }^{[1]}$. The result of the Tzanakis score and Yash score was reported independently. The result was correlated with the findings obtained on histopathological examination and the data was analyzed using the necessary statistical calculations using SPSS 24.0 version, the results were then presented.

\section{Observations and Results}

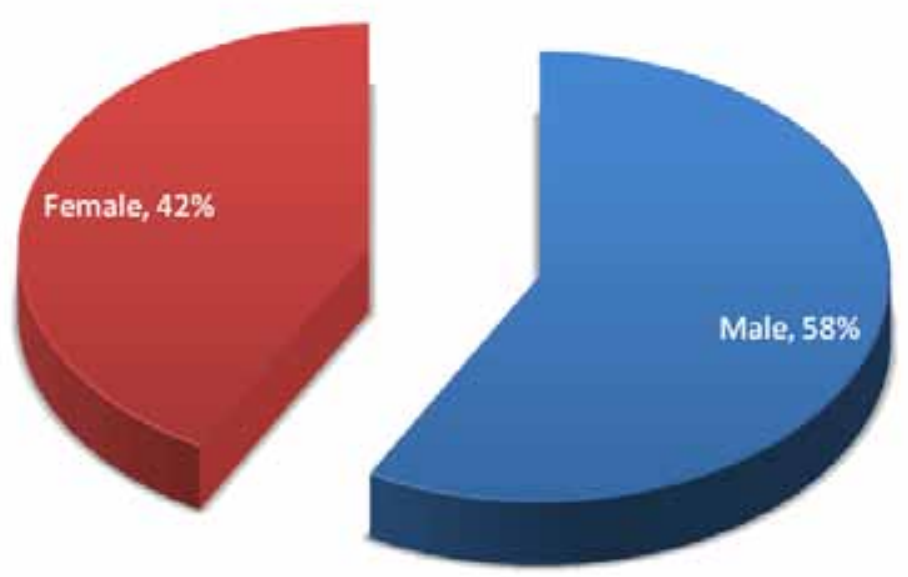

Graph No. 01: Gender distribution

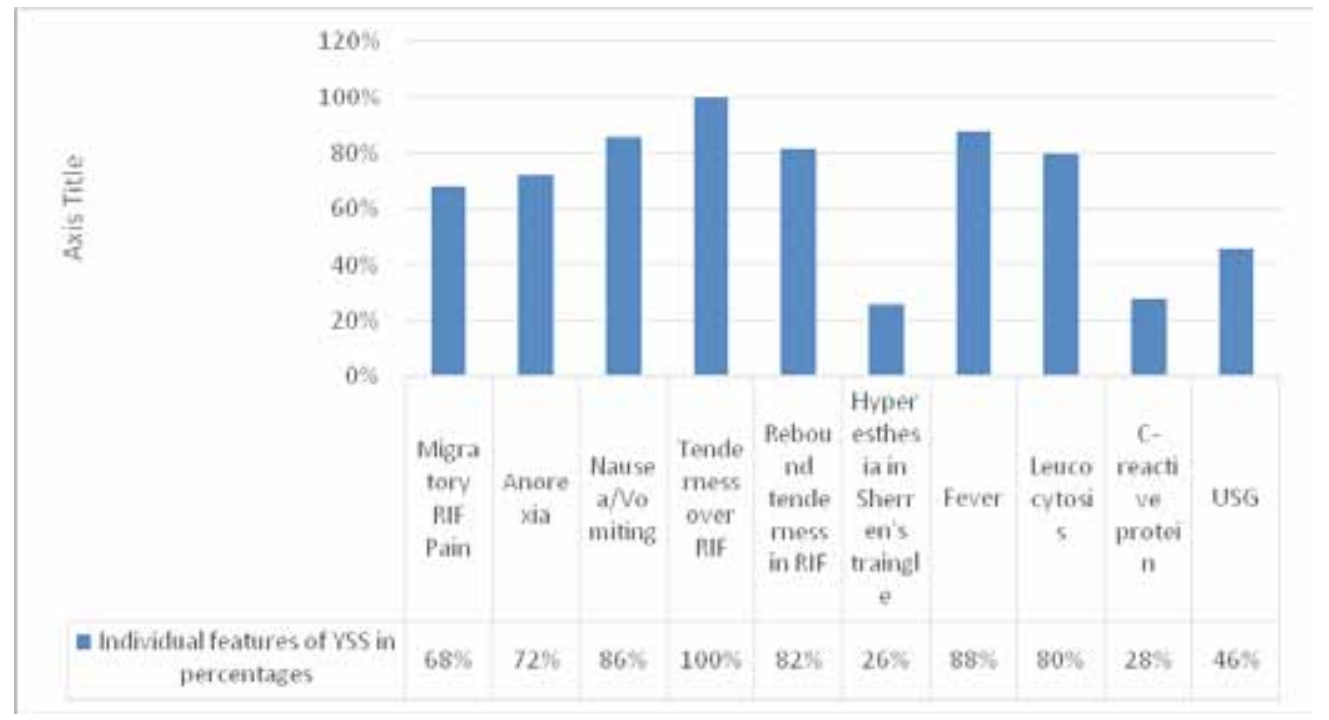

Graph 02: Characteristics of Yash score 


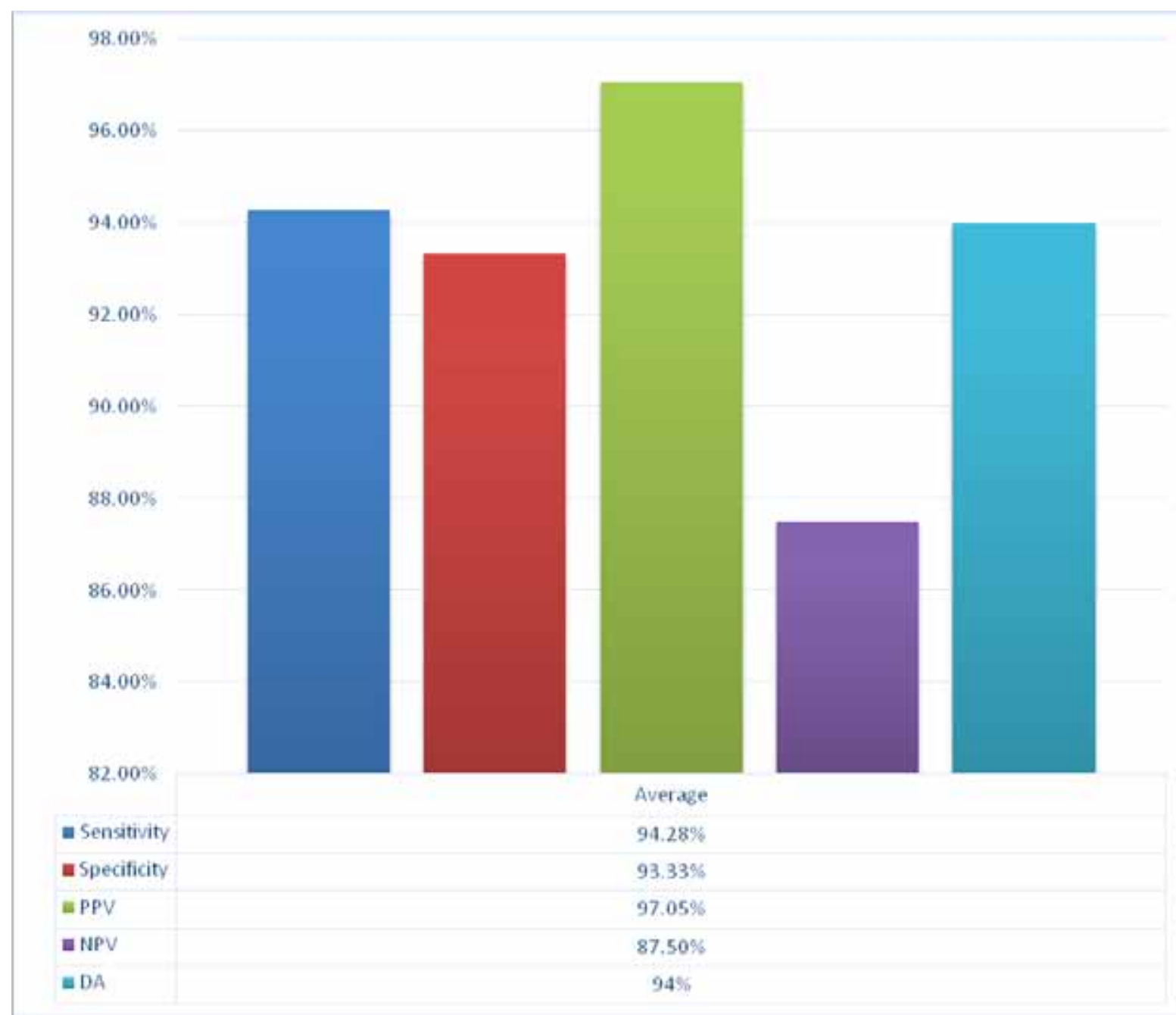

Graph 03: Outcome of YASH scoring system

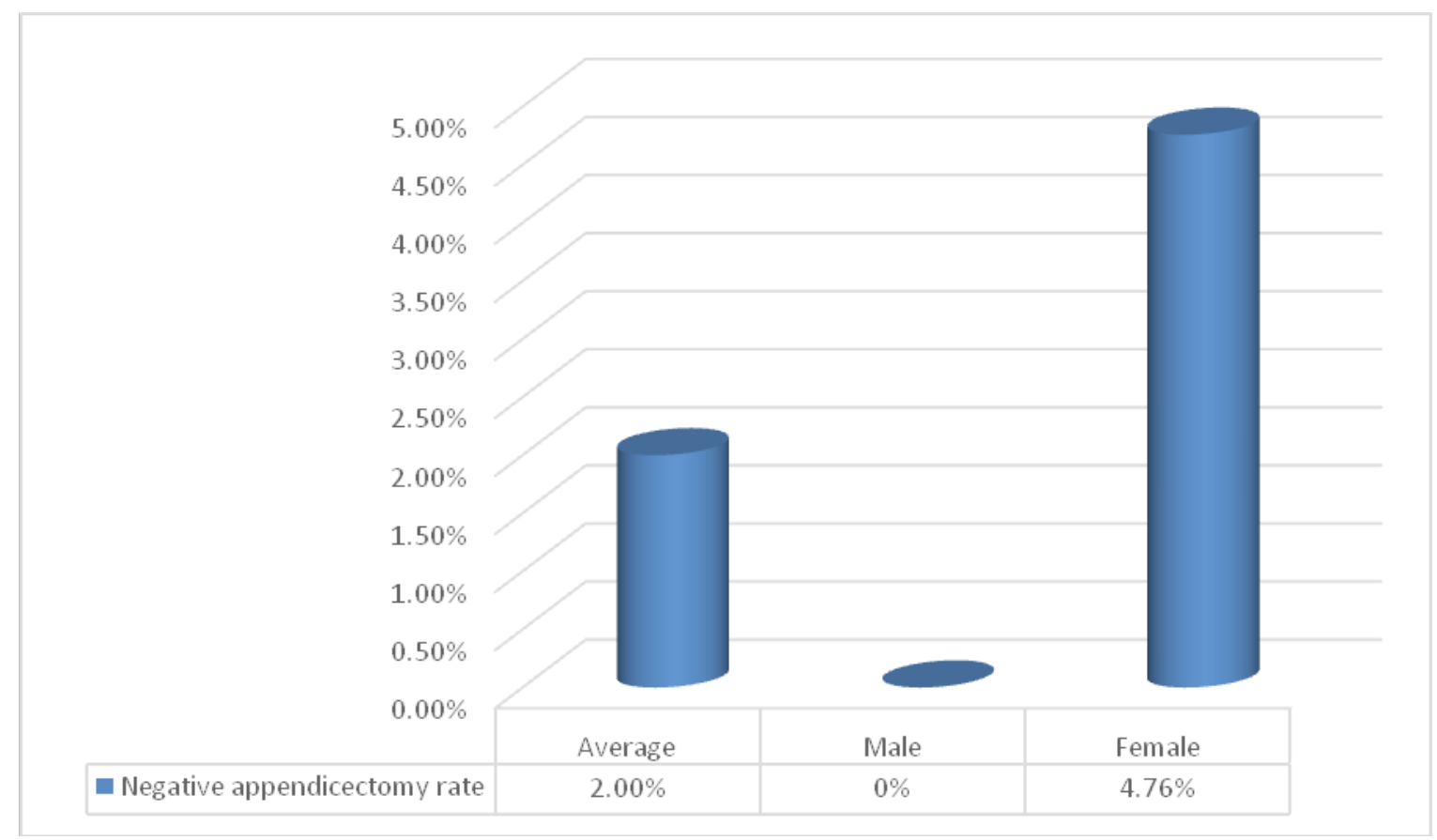

Graph 04: Negative Appendectomy rate of Yash scoring system: 


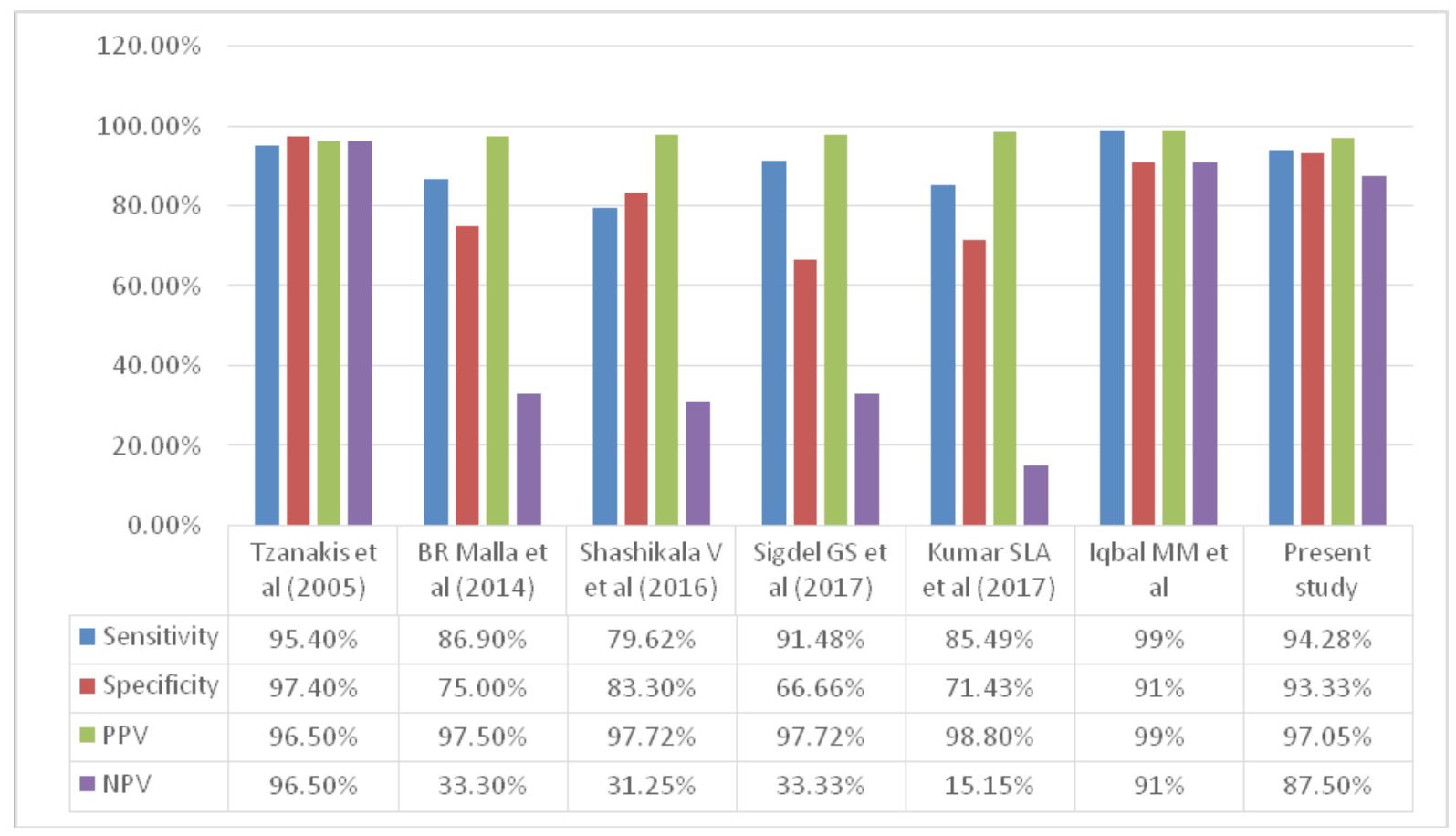

Graph 5: Comparison with various studies

\section{Discussion}

Acute appendicitis continues to exist as the most widespread emergency in the world. A delay in making a diagnosis is associated with various complications which increases the morbidity and mortality in patients therefore a prompt and reliable diagnosis of acute appendicitis is mandatory. Further more, negative appendicectomy also accounts for the loss of financial resources and is associated with morbidity in 10 to $15 \%$ of cases.

Despite big breakthroughs in the imaging field, there remains uncertainty in diagnosing acute appendicitis due to atypical presentations of the disease. It has been repeatedly shown that investigations like USG lack specificity due to its operator dependency whereas investigations like CT scan and MRI are highly-priced demand more advanced equipment and competency. This makes a detailed clinical examination with primary investigations such as leucocyte count as the backbone in diagnosing acute appendicitis. This has compelled many surgeons to use diverse scoring systems for diagnosing acute appendicitis. The clinical evaluation is reliable in $50 \%$ to $80 \%$ of cases. The evaluation is more complex in the extremes of age and women of reproductive age group due to atypical presentations.
The current study will deal with the evaluation of the Yash scoring system to diagnose acute appendicitis in a simple, reliable, and cost-effective way thereby reducing the negative appendectomy rate and thus morbidity associated with it.

In this study, the sample population consisted of 50 patients out of which $58 \%$ were males and $42 \%$ were females (see graph 01).

All the patients who accomplished the eligibility criteria were subjected to detailed clinical examination, routine hematological investigations, C-reactive protein, USG and were scored based onthe Yash scoring system. The decision to operate the patient including patients with scores less than the cut-off value was based on the clinical assessment and judgment taken by the consultant surgeon. All the patients underwent appendectomy with prior consent and the specimen was subjected to histopathological examination to confirm the diagnosis.

In our study, $82 \%$ of the patients were operated by open method whereas $18 \%$ of patients were operated by laparoscopy. The study done by Malla BR et a ${ }^{[14]}$ consisted of 200 patients in which 128 patients $(64 \%)$ underwent appendectomy by an open method and 72 patients (36\%) underwent appendectomy by laparoscopy. 
The most common incision used in open appendectomy was Mc Burney's in about $83 \%$ of cases followed by the right Para median which was used in $17 \%$ of cases. Similarly, in the study done by Lamture YR et al ${ }^{[12]}$ the most common incision used was Mc Burney's in 94.74\% of patients followed by right Para median in 5.26\% of cases. In our present study, 35 out of 50 patients $(70 \%)$ had inflamed appendix intra-operatively. Out of the remaining 15 patients, five patients had enterocolitis, three patients had Meckel's diverticulitis, three patients had the pelvic inflammatory disease (salpingitis), two patients had a ruptured ovarian cyst and two patients had inflamed mesenteric lymph node (with or without pus) as the intra-operative findings. Shashikala $\mathrm{V}$ et al ${ }^{[9]}$ in her study had five out of 50 patients with the alternative diagnosis; out of which one patient had enterocolitis and four patients had the pelvic inflammatory disease. Another study was done by Kumar SLA et al ${ }^{[13]}$ his study also reported six patients with alternative diagnoses in which three patients had salpingitis, two patients had an ovarian cyst and one patient had Meckel's diverticulitis.

The new Yash score described by Lamture YR et $\mathrm{al}^{[12]}$ in 2017 has a significant role in identifying acute appendicitis. It differs from earlier scoring systems by including various parameters such as C-reactive protein, leukocyte counts, USG, and clinical data. A score of seven or more in patients was considered as acute appendicitis and such patients were subjected to operative intervention.

In our study, out of ten parameters, tenderness in the right iliac fossa was the commonest sign seen in $100 \%$ of patients. The other two signs i.e. rebound tenderness and hyperesthesia in Sherren's triangle was seen in $82 \%$ and $26 \%$ of patients respectively. The most common symptom was fever which was present in $88 \%$ of cases followed by nausea or vomiting which was found in $86 \%$ of the cases. Anorexia was seen in $72 \%$ of patients, whereas $68 \%$ of patients gave a history of migratory right iliac fossa pain. Out of the three investigations included in the scoring system, leucocytosis defined as $\mathrm{WBC}$ count more than $10000 / \mathrm{mm}^{3}$ was present in $80 \%$ of cases. C-reactive protein with a value of more than $15 \mathrm{mg} / \mathrm{dl}$ was present in $28 \%$ of cases with USG showing features of appendicitis in only $46 \%$ of cases (see graph 02).

In the present study, 34 patients had a score of seven or more, and16 patients who had a score of less than seven according to the Yash score. Out of the 34 patients who scored seven or more, there were 33 patients with features of appendicitis on histopathological examination whereas only one patient hada histologically normal appendix with no features of inflammation. Similarly, out of 16 patients who scored less than seven, there were 14 patients with a histologically normal appendix with no features of inflammation and only two patients with features of appendicitis on histopathology.

The sensitivity and specificity of the Yash scoring system in the present study were found to be $94.28 \%$ and $93.33 \%$ respectively. It had PPV and NPV of $97.05 \%$ and $88.50 \%$ respectively. The overall diagnostic accuracy of the Yash scoring system in our study was found to be $94 \%$ (see graph 03 ). The following results are comparable to the single original study done by Lamture YR et al in which the sensitivity, specificity, PPV, and NPV was $99.48 \%, 92.86 \%, 99.48 \%$, and $92.85 \%$ respectively. The diagnostic accuracy of the Yash score reported by Lamture YR et al was $98.56 \%$.

The negative appendicectomy rate (NAR) of the Yash scoring system in the present study was found to be zero percent in males and $4.76 \%$ in females. This discrepancy in NAR was due to the high probability of another possible diagnosis in females of reproductive age group such as pelvic inflammatory diseases and ovarian cyst. The overall NAR observed for the Yash scoring system was $2 \%$ (see graph 04 ) which is way lower than the accepted rate of 15 to $25 \%$. In the study done by Lamture YR et al ${ }^{[12]}$, the NAR was found to be $6.69 \%$ which is comparable to our study ${ }^{[14]}$.

The negative appendectomy rate of the Yash scoring system was dramatically lower than other studiesand the diagnostic accuracy of this scoring system is better when compared with other studies (see graph no 5).

\section{Conclusion}

The study also shows that the Yash scoring system is a better tool to avoid unnecessary operations due to its exceptionally low negative appendectomy rate thus lowering morbidity in patients of acute appendicitis and thereby lowering the cost of treatment and prevention of misuse of valuable resources and manpower.

Ethical Clearance: Taken from institutional ethics committee.

Source of Funding: Self.

Conflict of Interest: Nil. 


\section{References}

1. Lamture $\mathrm{Y}$ et al. anatomical variations related to position of appendix.journal of evolution of medical and dental sciences-JEMDS (2018)7 (46), 5030-5033.

2. Vaidya $\mathrm{V}$ et al. Reliability of leukocytosis in diagnosing acute appendicitis. J. Evolution Med. Dent. Sci. 2020;9(32):2274-2278.

3. Vaidya $\mathrm{V}$ et al. Migration of abdominal pain - an effective tool to identify appendicitis and the only parameter to screen. J Evolution Med Dent Sci 2020;9(33):2329-2333,

4. MundadaA et al. Anorexia in acute appendicitis: A non-specific factor with significant accuracy in diagnosis. Medical Science, 2020, 24(105), 28122816

5. Grönroos J, Grönroos P. Leucocyte count, and C-reactive protein in the diagnosis of acute appendicitis. Br J Surg. 1999; 86(4):501-504.

6. Birnbaum B, Wilson S. Appendicitis at the millennium. Radiology. 2000; 215(2):337-348.

7. Khanal B et al. Accuracy of ultrasonography in the diagnosis of acute appendicitis. Kathmandu Univ Med J (KUMJ). 2008;6(1):70-74.

8. Tzanakis $\mathrm{N}$ et al. A new approach to accurate diagnosis of acute appendicitis. World J Surg. 2005;29(9):1151-56.
9. Shashikala V. Comparative study of Tzanakis score vs Alvarado score in the effective diagnosis of acute appendicitis. Int $\mathrm{J}$ Biomed Adv Res. 2016;7(9):41820.

10. Iqbal $\mathrm{M}$ et al. Experience of Tzanakis Scoring System for Accurate Diagnosis of Acute Appendicitis in Jinnah Postgraduate Medical Centre, Karachi. Isra Med J. 2018; 10(1): 40-43.

11. Sigdel G, Lakhey P, Mishra P. Tzanakis score vs Alvarado score in acute appendicitis. Department of Surgery, Kathmandu Model Hospital, Nepal, Department of surgery, Tribhuvan University Teaching Hospital, Kathmandu, Nepal. J Nepal Med Assoc. 2010;49(178):96-9.

12. Lamture $Y$ et al. Clinicosonological and laboratory co-relation with histopathology of acute appendicitis to develop new diagnostic scoring system (Yash scoring system). Int Surg J. 2017;4:2556-64.

13. Kumar $\mathrm{S}$ et al. Evaluation of Tzanakis scoring system in acute appendicitis: a prospective study. IntSurg J. 2017;4:3338-43.

14. Malla B et al. Comparison of Tzanakis Score vs Alvarado Score in the Effective diagnosis of acute Appendicitis. Kathmandu Univ Med J. 2014;45(1):48- 50. 